CORRIGENDUM

doi:10.1038/nature09167

\title{
International network of cancer genome
} projects

The International Cancer Genome Consortium

Nature 464, 993-998 (2010)

In this Perspective, author Heinz Himmelbauer was incorrectly listed as Heinz Himmelbaue, author Brooke B. Gardiner was incorrectly listed as Brooke A. Gardiner, and author Anthony Cros was incorrectly listed as Anthony Cross. 